\title{
The BCS annual conference, Manchester, 2-4 June 2014: The vice-president's message
}

\author{
Sarah Clarke
}

The 2014 conference will host our most extensive programme of education to date. In addition, this year we anticipate that over 3000 delegates will attend, as the British Heart Foundation (BHF) Health Care Professionals are also joining us. However, it is not just about size of the event. Quality is key, and we strive to deliver high-quality education with experts and key opinion leaders from the UK and abroad.

\section{HIGHLIGHTS OF THE PROGRAMME}

This year our theme is 'Tomorrow's World' and many of the plenary sessions will be looking to the future of cardiovascular science and medicine. The conference will be opened by the President, Dr Iain Simpson, with an opening lecture delivered by Professor Greg Whyte.

The main programme is divided into dedicated tracks, as follows:

- National training day: for trainees and includes a session with the Specialist Advisory Committee

- Education for revalidation: covering topics from the cardiology curriculum over a 5-year cycle to facilitate revalidation

- Affiliated groups: with an expanded number of dedicated sessions, joint sessions and dedicated tracks. This year we welcome the British Hypertension Society to the meeting

- Imaging: sessions spread over 2 days and covering multi-modality imaging with a 'read with the experts session' and a poster session too

- Clinical science translational research: looking to the future, this track also includes the Young Research Workers' Prize (YRWP), Michael Davies Early Career Award, and poster sessions

- Basic science: the British Atherosclerosis Society (BAS)/British Society for Cardiovascular Research (BSCR) Spring Meeting includes the Young Investigator

Correspondence to Dr Sarah Clarke, Department of Cardiology, Papworth Hospital, Lakeside Crescent, Papworth Hospital, Cambridge CB23 3RE, UK; clarkes@bcs.com
Award (YIA) and basic science poster sessions

- British Cardiovascular Society (BCS) sessions: developed by the programme committee, we repeat some old favourites (eg, top 10 trials, a live multidisciplinary team (MDT) meeting with coronary and structural cases, the cardiology quiz), but introduce new sessions including JBS3 Joint British Societies for Prevention of Cardiovascular Disease-report highlighting the new 'JBS3 risk calculator' (see April supplement Heart 100 suppl 2), cardiology 'rocks' (Mark Richardson from Skunk Anansie will undertake CPEX testing while on the drums), and cardiac physiology at the top of Everest (with a climber who has been there eight times!)

- Joint sessions with the European Society of Cardiology (ESC) and American Society of Cardiology (ACC) and, new this year, joint sessions with an expert panel from the Mayo Clinic

- Lifelong learning: our new revalidation initiative. This is led by Professor Simon Ray. The UK is not alone in requiring doctors to demonstrate knowledge of contemporary evidence and practice across the breadth of their practice. In the USA, a process of maintenance of competence (MOC) is being introduced, which requires similar completion of continuing professional development (CPD) but also an examination. The ACC and the Mayo Clinic have been at the forefront of developing innovative interactive educational courses to support MOC. This year for the first time, the BCS will be running two of these interactive educational sessions at the conference. The sessions have been developed in partnership with the ACC and are specifically designed to review recent developments across the spectrum of general cardiology to support revalidation. Each session will last for 2.5 hours and will consist of 30 clinical scenarios with accompanying multiple choice questions, which the audience will answer using response pads. Key to the success of these sessions is the moderators who introduce each scenario and discuss the responses with the audience, with immediate reference to recent evidence and guidelines. We are extremely fortunate to have as moderators in Manchester two outstanding teachers from the Mayo Clinic, Dr Rick Nishimura and Dr Carole Warnes. Places for the interactive sessions will be limited and will be allocated on a first come first served basis via registration on the BCS website http://www.bcs.com

- Named lectures from Dr Rick Nishimura (pericardial disease: a new look at an old disease) and Professor Barbara Casadei (a radical view of atrial fibrillation)

- BHF Healthcare Professionals: a new linked day for 2014

- Sponsored symposia: including a competition to find the most innovative pathway for heart failure and atrial fibrillation

\section{EDUCATION HALL}

The 'Education Hall' is now a dedicated area for 'interactive' education provided by the BCS, BHF and industry colleagues:

- Hot topics: these are short $15 \mathrm{~min}$ presentations of key messages and 'How to...' sessions covering over 110 topics. There are also interviews with some of the faculty. Hosted in four zones, one of the zones is run by the BHF

- Simulators: open to all technical skills, simulation is provided on a wide array of simulators (including angiography, percutaneous coronary intervention, transoesophageal echocardiography, transcatheter aortic valve implantation, renal denervation and pericardocentesis). A certificate will be issued on satisfactory completion of objectives

- Imaging village: interactive work stations will be available for CT, MRI, nuclear medicine and echo. There will be 'live' echo scanning. An MRI scanner will be on site and scanning of volunteers will facilitate learning postprocessing skills and scan interpretation

- Resuscitation skills: the Resuscitation Council are providing updates and resuscitation skills training. Certificates will be issued on completion. Sign up alone or as a team

- Industry stands: an education agenda is provided by our industry colleagues on the stands and we thank them for their support

- Poster area: the Education Hall hosts the abstract/poster sessions for interactive discussion with lead authors, 
facilitated by discussants. Over 250 posters will be presented

While the Education Hall is a great place to learn and interact with colleagues and industry, our annual dinner also provides an excellent forum for networking.

The full programme, additional information about the conference sessions, and booking for lifelong learning, simulation, imaging village, resuscitation skills sessions and the annual dinner are available on line at http://www.bcs.com/annualconference. A microsite is available for mobile phones and also an App including biographies of the faculty. Many sessions will be webcast. Podcasts will be produced in conjunction with the BMJ. Follow us on twitter @BritishCardioSo or use \#bcs2014. We are also on Facebook.

On behalf of the BCS President and the Programme Committee for the Annual Conference 2014, I welcome you to Manchester and what will be a highquality educational programme in both plenary and interactive sessions- something for everyone in a dedicated environment. We hope you have an enjoyable and rewarding time with us!
Competing interests None.

Provenance and peer review Commissioned; internally peer reviewed.

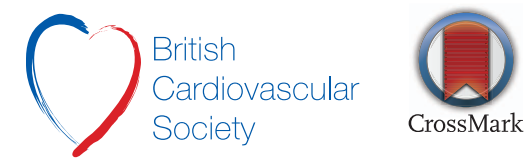

To cite Clarke S. Heart 2014;100:743-744.

Accepted 24 March 2014

Heart 2014;100:743-744.

doi:10.1136/heartjnl-2014-305918 